ISSN 2179-6750

\title{
Acometimento da Síndrome de Burnout em enfermeiros da atenção básica e o impacto na gestão do serviço
}

Involvement of Burnout Syndrome in nurses of primary care and the impact in management services

La Síndrome de Burnout en enfermeros de la atención primaria y el impacto en la gestión de los servicios

Gabriela Kurzawski Nunes ${ }^{1}$; Sonia Maria Cano ${ }^{2}$; William Malagutti ${ }^{3}$

\section{Resumo}

Mudanças no âmbito do trabalho decorrentes do processo de globalização atreladas ao aumento da competitividade e expansão tecnológica, sobretudo a partir dos anos 1990, configuraram um contexto de grande pressão sobre os profissionais em diversas áreas de atuação. Os trabalhadores respondem de maneira cada vez mais negativa a esse ambiente competitivo, e muitas vezes aparecem suscetíveis aos vários tipos de doenças mentais, como a Síndrome de Burnout. Nesse contexto, encontram-se os profissionais de saúde. Portanto, o objetivo deste trabalho é o de descrever e analisar o desencadeamento da Síndrome de Burnout em Enfermeiros nos diversos contextos de atuação. A metodologia utilizada neste trabalho foi o levantamento bibliográfico, com a seleção dos trabalhos mais pertinentes a temática, além da análise e articulação dos principais pontos identificados nesses trabalhos. Conclui-se com esse trabalho que são diversos fatores que colaboram para que o profissional desenvolva a síndrome desde: o ambiente de trabalho, as relações interpessoais (clientes, colegas e a instituição) e falta de investimentos que contribuam para a melhora do ambiente do profissional Enfermeiro. Por outro lado, contextos mais gerais como o ambiente socioeconômico hostil no qual se encontram muito dos pacientes, também aparecem como importantes fontes de tensão para o profissional Enfermeiro.

Descritores: Síndrome, Burnout, Enfermeiros, Atenção Básica

\begin{abstract}
Changes in scope of work resulting from the process of globalization linked the increased competitiveness and technological expansion, especially since the year 1990 configured hum context of high pressure over the professionals in several areas. Workers respond way every time more negative one this competitive environment, and often appear susceptible to various types of mental disorders, as Burnout Syndrome. Therefore, the objective is to describe and analyze the onset of Burnout Syndrome in nursesseveral contexts of practice. The methodology used in this work was the bibliographical survey, with the selection of more relevant work the theme, beyond the analysis and articulation of the main points identified sas work. Concludes with that workare several factors that collaborate to what the professionals develop a syndrome from: the work environment, such as interpersonal relations (clients, colleagues and the institution) lack of
\end{abstract}

\footnotetext{
${ }^{1}$ Administradora Hospitalar pelo Centro Universitário São Camilo. Discente do Curso de Especialização em Saúde Pública com ênfase na Estratégia de Saúde da Família do Centro Universitário São Camilo, São Paulo. Rua Cayowaa, 2175, Sumaré, São Paulo, SP, Brasil, CEP 01258011. email: gabrielaknunes@ gmail.com

${ }^{2}$ Administradora pela Universidade Anhanguera. Discente do Curso de Especialização em Saúde Pública com ênfase na Estratégia de Saúde da Família do Centro Universitário São Camilo, São Paulo. Rua Botafogo, 154, Vila Penteado, São Paulo, SP, Brasil, CEP 02864040. email: soniamcano@ hotmail.com

${ }^{3}$ Mestre em Administração, Comunicação e Educação pela Universidade São Marcos - São Paulo. Docente do Curso de Especialização em Saúde Pública com ênfase na Estratégia de Saúde da Família do Centro Universitário São Camilo,

São Paulo. Rua Raul Pompéia, 144, Pompeia, São Paulo, SP, CEP: 05025-010. email: williammalagutti2@ hotmail.com
} 122 
ISSN 2179-6750

investment contributing to an improvement of the nursing professional environment . On the other side, contexts more general as the hostile socioeconomic environment without qua are lot of pacientes, also appear how important power supplies for nurse professionals.

Key-words: Syndrome, Burnout, Nurses, Primary Health

\section{Resumen}

Los cambios en el trabajo vinculados a la globalización aumento de la competitividad y la expansión tecnológica, especialmente desde la década de 1990, configuran un entorno de alta presión de profesionales en diversos campos. Los trabajadores responden cada vez más negativo en este entorno competitivo, y con frecuencia parecen susceptibles a varios tipos de enfermedades mentales tales como el Síndrome de Burnout. Por lo tanto, el objetivo de este estudio es describir y analizar la aparición del síndrome de burnout en enfermeras en los diferentes contextos de acción. La metodología utilizada fue la revisión de la literatura, con la selección de los trabajos temáticos más relevantes, así como el análisis y la articulación de los principales puntos identificados en estos estudios. Se concluye con este trabajo que son varios los factores que contribuyen al profesional a desarrollar el síndrome de: el entorno de trabajo, las relaciones interpersonales (clientes, colegas y la institución) y la falta de inversión que contribuyen a la mejora del entorno profesional enfermera. Por otra parte, el contexto más general como el ambiente hostil socioeconómico en el que los pacientes estan parecen ser importantes fuentes de tensión a la enfermera.

Palabras-claves: Síndrome de Burnout, Enfermeras, Atención Primaria

\section{Introdução}

A Síndrome de Burnout enquanto problemática decorrente da relação entre trabalhador e seu ambiente de trabalho, se intensificou num contexto de complexas transformações no mundo do trabalho. Essas transformações estão relacionadas, de maneira geral, às mudanças políticas, sociais e técnicas do mundo atual, sobretudo, com o processo de globalização ${ }^{1}$. Podemos caracterizar a Síndrome de Burnout como consequência de "prolongados níveis de estresse no trabalho e compreende exaustão profissional, distanciamento das relações pessoais e diminuição do sentimento de realização profissional." ${ }^{2}$.

A Síndrome de Burnout está relacionada a todas as áreas profissionais, mas o objetivo deste trabalho se restringe a analisar o caso da síndrome em profissionais da saúde, mais especificamente, nos profissionais Enfermeiros. "Os enfermeiros fazem parte de uma profissão caracterizada por ter o cuidado como sua essência e por grande parte da carga de trabalho ser o contato direto com pacientes e familiares." 3 . Portanto, é importante que se estude essa questão, tendo em vista, os diferentes contextos nos quais ela está presente. A Síndrome de Burnout prejudica não apenas os profissionais Enfermeiros que sofrem com a doença em si, mas como a entidade em que eles atuam, seus colegas de trabalho e pacientes. Por este motivo o maior conhecimento da doença pode ajudar na prevenção, diagnóstico e tratamento. 
ISSN 2179-6750

No caso deste trabalho será analisada a Síndrome de Burnout nos profissionais Enfermeiros, baseando-se em diferentes experiências levantadas pela revisão bibliográfica. Dentre esses diferentes contextos, se destacam o caso da Síndrome de Burnout em Unidades Básicas de Saúde.

A pergunta central que norteia essa pesquisa é: quais as relações entre o acometimento da Síndrome de Burnout e o ambiente de trabalho e as atividades exercidas pelo enfermeiro? Para responder essa pergunta o objetivo deste trabalho é o de, a partir do levantamento bibliográfico, analisar o caso da Síndrome de Burnout em profissionais da saúde, mais especificamente, nos enfermeiros utilizando como referência diferentes contextos de atuação desses profissionais na Atenção Básica. Assim, este trabalho teve como objetivo geral descrever e analisar o desencadeamento da Síndrome de Burnout nos profissionais Enfermeiros em diversos contextos de atuação, particularizando Enfermeiros que atuam na Atenção Básica e como essa situação implica na Gestão e Qualidade dos Serviços de Saúde.

\section{Metodologia}

A metodologia utilizada para realização desse trabalho foi a revisão bibliográfica integrativa. Nesse sentido, foi feita a busca de artigos nas bases de dados MEDLINE, SciELO, LILACS e o endereço eletrônico da Organização Mundial da Saúde (OMS), utilizando como critério de seleção os artigos pertinentes aos seguintes descritores: Síndrome; Burnout; Enfermeiro; Atenção Básica. A busca foi feita para o período compreendido entre 2000 e 2015, para artigos publicados em língua latina considerando-se a sua atualidade; dissertações, teses e livros sem restrição temporal e de idioma, de acordo com a relevância do tema. A análise do material bibliográfico foi feita através de leitura exploratória que definiu quais textos se enquadravam na temática relacionada à Burnout, seus aspectos conceituais, o acometimento nos trabalhadores da área da saúde e suas conseqüências na gestão dos serviços. Em seguida, em uma leitura mais aprofundada dos textos selecionados, buscou-se localizar informações relacionadas ao objetivo deste estudo.

\section{Resultados e Discussão}

A preocupação acerca da Síndrome de Burnout tem ganhado relevância nas duas últimas décadas, as mudanças sociais e econômicas e o consequente aumento da competitividade no mundo do trabalho são aspectos mais gerais que contribuem para a ascensão da doença ${ }^{4}$. Nesse sentido, “as taxas de turnover e a escassez de enfermeiros capacitados no mercado tem contribuído para a mobilização de associações profissionais no sentido de sensibilizar os órgãos públicos e os gestores das instituições privadas para uma reavaliação da atuação do enfermeiro nos vários níveis de 
ISSN 2179-6750

atenção à saúde." ${ }^{4}$. No âmbito das instituições formais a Síndrome de Burnout apresenta a seguinte definição:

"No Brasil a Previdência Social reconhece o Burnout como agente patogênico e o Decreto no 3.048, de 06 de maio de 1999, aprovou o anexo II do Regulamento da Previdência Social que trata dos Agentes Patogênicos causadores de Doenças Profissionais o qual cita a "sensação de estar acabado." 4 .

No que se refere a abordagem acadêmica, a Síndrome de Burnout conhecida também como síndrome do esgotamento profissional "é um tipo de resposta prolongada a estressores emocionais e interpessoais crônicos no trabalho. Tem sido descrita como resultante da vivência profissional em um contexto de relações sociais complexas, envolvendo a representação que a pessoa tem de si e dos outros." 5 .

A Síndrome de Burnout apresentam três elementos centrais:

- $\quad$ exaustão emocional (sentimentos de desgaste emocional e esvaziamento afetivo);

- despersonalização (reação negativa, insensibilidade ou afastamento excessivo do público que deveria receber os serviços ou cuidados do paciente);

- diminuição do envolvimento pessoal no trabalho (sentimento de diminuição de competência e de sucesso no trabalho)" 5 .

É importante que se diferencie a Síndrome de Burnout do quadro tradicional de estresse. O quadro tradicional de estresse não necessariamente se refere ao esgotamento físico e psicológico advindo com a prática profissional, já a Síndrome de Burnout "envolve atitudes e condutas negativas com relação aos usuários, aos clientes, à organização e ao trabalho, sendo uma experiência subjetiva que acarreta prejuízos e práticas emocionais para o trabalhador e a organização" 5 .

Abordagem geral da temática

As mudanças no mundo do trabalho advindas com o processo de globalização, intensificado a partir dos 1990, impactaram de maneira importante os países subdesenvolvidos que se integram de maneira passiva nesse processo. A abertura dos mercados e o aumento da competição impactaram fortemente na vida dos trabalhadores ${ }^{6}$. Nesse sentido, "como consequência da velocidade das mudanças no mundo do trabalho e da dificuldade de adaptação do ser humano a essas mudanças, surgem reações como insatisfação generalizada com o modo de vida, tédio, angústia, ambiguidades, ansiedade, despersonalização, frustração e alienação no trabalho, entre outros." 7 . Portanto, a importância dos estudos que analisam doenças no âmbito do trabalho residem 
ISSN 2179-6750

na possibilidade de: "elaboração e implementação de estratégias que possam contribuir na melhoria da qualidade de vida no cotidiano profissional do Enfermeiro, tornando o cuidar/cuidado uma via de mão dupla na relação profissional X cliente X organização de saúde.” 7 .

Tendo esse aspecto de maneira mais geral, citado acima, em mente, essa seção procura analisar as causas da Síndrome de Burnout em Enfermeiros nos diferentes contextos de atuação do profissional da área. Dentre os fatores mais gerais que contribuem para desencadeamento da doença esses autores apontam o número reduzido de profissionais de enfermagem em proporção à demanda, excesso em quantidade e variedade de atividades a serem conciliadas e executadas, dificuldade em delimitar os diferentes papéis entre as variadas classes da enfermagem, falta de reconhecimento do público assistido e dos próprios colegas, do grupo multidisciplinar e ainda da organização ${ }^{7}$.

Os autores continuam, apontando que as relações no trabalho que contribuem ao desenvolvimento da doença "abrange todo tipo de relações interpessoais, desde cliente/paciente, passando por colegas de trabalho, até chefes/supervisores. O trabalhador perde o sentido de sua relação com o trabalho, as coisas já não têm mais importância e todo esforço parece ser inútil." 7 .

Outros autores vão apontar a relação da Síndrome de Burnout com a prática do Enfermeiro entendendo que, por sua própria natureza e características de seu trabalho, revela-se suscetível ao fenômeno do estresse ocupacional em decorrência da responsabilidade pela vida e a proximidade com os clientes para os quais o sofrimento é quase inevitável. "Exige-se destes profissionais a dedicação no desempenho de suas funções, o que aumenta a possibilidade de ocorrência de desgastes emocionais em altos níveis de estresse, tornando-os vulneráveis à cronificação do estresse ocupacional que se denomina de Síndrome de Burnout." 8.

Elencamos aqui alguns fatores que remetem à adversidade na ocupação do profissional Enfermeiro que atua na Atenção Primária à Saúde:

a) Grande número de pacientes a serem atendidos em diferentes contextos de suas patologias, em diferentes faixas etárias, que necessitam de um olhar muito técnico e assertivo dos Enfermeiros para um atendimento humanizado e eficaz;

b) O baixo nível de adesão de pacientes, fazendo com que os Enfermeiros tenham que organizar o fluxo de Visitas Domiciliares com maior frequência, sobrecarregando-os em suas tarefas já existentes;

c) A formação inadequada de profissionais que estão sendo inseridas no mercado de trabalho, remetendo à ruídos de comunicação entre a própria 
equipe de trabalho, condutas errôneas direcionadas aos pacientes, comprometendo a qualidade de assistência prestada e expondo-os a um nível de segurança duvidosa;

d) Falta de RH em número adequado na equipe de Enfermagem, em relação à grande contingente de pacientes a serem assistidos;

e) Desvio de função do profissional Enfermeiro, que acaba sendo utilizado em Recepção, Farmácia e Laboratórios entre outros setores, causando maior sobrecarga de tarefas em seu cotidiano de trabalho;

f) Grande complexidade e diversidade de tarefas direcionadas aos Enfermeiros de algumas Organizações Sociais, que tem como objetivo metas de produção;

g) Violência institucional exacerbada em alguns equipamentos de saúde, devido ao perfil da clientela a ser atendida, principalmente, em locais de periferias das grandes cidades, sejam por falta de educação, formação ou respeito, bem como devido falta de profissionais médicos que dêem conta da demanda de atendimento, e como o Enfermeiro faz o Acolhimento, este acaba sendo a "válvula de escape" dos desafetos desta população.

Todos esses fatores supracitados denotam uma falta de planejamento estratégico dos serviços de saúde, que acabam remetendo a falhas estruturais na organização do trabalho, afetam os profissionais de saúde e repercutem na qualidade da assistência prestada aos clientes e usuários da Atenção Básica.

\section{A síndrome em diferentes contextos}

O estudo identificou que a ocorrência da Síndrome de Burnout está ligada a fatores que eles vão definir como internos e externos ao trabalho. No que se refere aos fatores internos, esses estão relacionados a processos como: dinâmicas de horário (turno do trabalho), o caráter do serviço prestado e o local de trabalho, além das relações interpessoais e falta de estrutura. Já os fatores externos estariam ligados ao perfil psicossocial do profissional, que incluem pontos como: sexo, idade, renda e hereditariedade ${ }^{7}$.

A seguir será apresentada a análise de alguns estudos identificando os principais pontos fatores internos e externos - que compreende a relação Síndrome de Burnout e prática do Enfermeiro. Os contextos de atuação os quais serão analisados são: Estratégia de Saúde da Família e Unidades Básicas de Saúde.

No caso da Síndrome de Burnout que acometem profissionais Enfermeiros atuantes no 127 
ISSN 2179-6750

programa de Estratégia da Saúde da Família, as principais causas para o desencadeamento da síndrome se relacionam, de maneira geral, a ausência de recursos para que os Enfermeiros possam atender as demandas do trabalho ${ }^{1}$. Acrescentando-se a precariedade de recursos que o profissional dispõe "os membros da ESF também se deparam com ambientes, muitas vezes, perigosos, insalubres e propícios a riscos à saúde, o que se adiciona às pressões e exigências do próprio trabalho e favorece o desenvolvimento da Síndrome de Burnout e outras doenças relacionadas ao trabalho" 1 . Na amostragem estudada por esses autores identifica-se que a prevalência desta síndrome está entre trabalhadores jovens, sendo mais comum entre os que ainda não alcançaram 30 anos; e é atribuída a pouca experiência do trabalhador, a qual acarreta insegurança, ou choque com a realidade quando este percebe que o trabalho não garantirá a realização de suas ansiedades e desejos. A idealização, comum entre jovens trabalhadores, associa-se a expectativas elevadas, que muitas vezes não são concretizadas. Logo, a excessiva motivação, paradoxalmente, torna os indivíduos mais vulneráveis à Síndrome de Burnout. Na presente pesquisa verificou-se que o grupo mais jovem, com idade entre 20 e 40 anos obtiveram escores com diferença estatística significativa em desgaste emocional e despersonalização, quando comparados com outras faixas etárias ${ }^{1}$.

A ocorrência da síndrome está diretamente relacionada ao modo de operação do programa do Sistema Único de Saúde, ao iniciar na ESF, é recomendado que os trabalhadores recebam treinamento para atuarem em consonância com esta modalidade de atenção do Sistema Único de Saúde (SUS). O perfil inadequado dos trabalhadores, o exacerbo de cooperativismos, a falta de capacitação, a remuneração insuficiente e a ausência de uma política de recursos humanos, além de impedirem a construção de um sistema de saúde universal, integral e equânime, podem gerar frustração profissional ao trabalhador ${ }^{1}$.

Por outro lado, a Síndrome de Burnout relacionado aos profissionais Enfermeiros que atuam em Unidades Básicas de Saúde tem como principais fatores de desencadeamento da síndrome a "luta por reconhecimento social, sobrecarga de trabalho, achatamento de salários, múltiplos vínculos empregatícios, recursos inadequados, contato direto com a dor e o sofrimento" 9.

O desencadeamento da síndrome no âmbito das Unidades Básicas de Saúde é apontado de maneira específica. Os autores através da coleta de dados e por meio da análise qualitativa elencaram algumas das queixas dos profissionais que foram mais recorrentes:
- $\quad$ Baixa remuneração;
- $\quad$ Poucos profissionais;
- $\quad$ Falta de materiais;
- $\quad$ Estrutura física inadequada; 
- $\quad$ Desgaste no trabalho;

- $\quad$ Falta de materiais para os usuários ${ }^{9}$.

O estudo aponta que as condições referentes a infraestrutura, e sobretudo, a questão da remuneração aparecem de forma mais intensa nas falas dos profissionais. Nesse sentido, "tal repetição pode demonstrar a maior preocupação por parte dos profissionais com os aspectos estruturais presentes no trabalho do Enfermeiro em unidades do sistema público de saúde, além de apresentar a possibilidade de nesse grupo de elementos estarem os possíveis fatores desencadeantes da síndrome" ${ }^{9}$. No entanto, o desencadeamento da síndrome em Unidade Básica de Saúde foi considerado baixo em relação a literatura estudada, mas como atenta os autores estes índices não podem ser ignorados pois influem no desempenho negativo do profissional ${ }^{9}$.

Na Atenção Básica esses autores apontam causas diferentes, nesse sentido, o estudo atribui o aumento das possibilidades do desencadeamento da síndrome nos profissionais, diante o contexto condições socioeconômicas que permeiam a vida dos pacientes e que exigem do profissional da saúde competências que, muitas vezes, extrapolam a formação do profissional.

Nas Unidades Básicas de Saúde sua implantação enfrenta um desafio adicional às usuais dificuldades de perfil de atuação do Enfermeiro, em função da complexidade da dinâmica da vida nas comunidades e bairros, com seus diversos agenciamentos econômicos e políticos, e pela mobilidade das vagas de trabalho, ao sabor dos investimentos governamentais municipais, determinando intenso fluxo migratório de profissionais entre as cidades, em especial nas áreas metropolitanas ao redor das capitais estaduais" 3 .

Entendemos que a eficácia da intervenção no combate à Síndrome de Burnout estabelece-se com maior resolutividade quando são empregadas estratégias com abordagem individual somadas por estratégias organizacionais.

Ações preventivas serão eficazes quando a síndrome não for estigmatizada unicamente como responsabilidade individual ou pelo relacionamento profissional-paciente, e começar a ser entendida com um problema de relação indivíduo-processo de trabalho-organização.

Há outros fatores que remetem os Enfermeiros que atuam na Atenção Básica ao estresse diário e muitas vezes desencadeando o Burnout :

- $\quad$ Quantidade de pacientes a serem atendidos com diversidade de patologias;

- A dificuldade de comunicação em pacientes com baixo nível cognitivo e sociocultural;

- $\quad$ Outros pacientes e/ou familiares hostis e/ou reivindicadores; 
Pacientes com alteração comportamental ou que estejam sob o efeito de drogas ilícitas;

- $\quad$ Os dilemas éticos na tomada de decisão no cotidiano de trabalho;

- As dificuldades no relacionamento interpessoal com sua equipe de trabalho e/ou multidisciplinar;

- $\quad$ A pressão da Chefia na resolutividade de tarefas e quantidade de atividades quando se tratar de Organizações Sociais, que tem uma meta mensal a ser cumprida.

Algumas considerações sobre o enfrentamento da questão

Tendo em vista o conteúdo apontado no desenvolvimento deste trabalho acerca das causas mais recorrentes para o desencadeamento da Síndrome de Burnout em diferentes contextos, é importante que algumas considerações sejam feitas no que cerne ao enfrentamento da doença.

A importância do cuidado do Enfermeiro tem sido demonstrada para todas as situações em que o ser humano tem agravos da saúde. Por isso, o Enfermeiro precisa cuidar de si para cuidar do outro. As condições de trabalho dos Enfermeiros, cada vez mais, vem sendo contempladas como objetos de pesquisa, devido aos riscos que o ambiente oferece para o trabalhador e para os pacientes e aos aspectos penosos das atividades peculiares ao cuidado de enfermagem ${ }^{10}$.

Quanto ao enfrentamento dos agentes estressores que levam à Síndrome de Burnout profissionais já diagnosticados, as organizações podem promover ações educativas sobre as possíveis formas de enfrentamento e viáveis mudanças nas condições do trabalho, lotação de funcionários conforme aptidão e afinidade com as diversas áreas do cuidar, e estimular em parceria com instituições de ensino superior o desenvolvimento de ações em educação permanente e continuada, além de pesquisas que permeiem os processos de gerir pessoas e talentos, elucidando possibilidades para redução desse fenômeno, considerado hoje de cunho sociorganizacional ${ }^{7}$.

As propostas de enfrentamento devem ser elaboradas de acordo com a necessidade individual de cada acometido pela síndrome, assim como devem ser feitos ajustes ambientais para a redução dos eventos adversos a nível organizacional e melhora da resposta do indivíduo ao ambiente de trabalho. Desta forma entendemos que o enfrentamento da Síndrome de Burnout não depende de uma única dimensão a ser vencida, e sim de mudanças multifatoriais. 


\section{Conclusão}

A título de conclusão cabe primeiramente apontar que o aumento da competitividade provocou maiores exigências relativas à qualificação do profissional da saúde, configurando um contexto no trabalho cada vez mais desgastante para esse profissional.

De maneira geral, com a realização do trabalho pôde-se concluir que são diversos fatores que colaboram para que o profissional desenvolva a síndrome desde: o ambiente de trabalho, as relações interpessoais (clientes, colegas e a instituição) e falta de investimentos que contribuam para a melhora do ambiente do profissional do Enfermeiro. A percepção por parte dos profissionais da ausência de investimentos que contribuam para a melhoria efetiva das suas condições de trabalho, por muitas vezes são fontes de tensão que contribuem de maneira importante para o desencadeamento da síndrome.

Por outro lado, contextos mais gerais como o ambiente socioeconômico hostil, no qual se encontram muito dos pacientes alvos da Atenção Básica, também aparecem como importantes fontes de tensão para o profissional Enfermeiro. Somado a isso, as condições de remuneração bem como de planos de carreira são motivações que se encontram associadas ao desencadeamento da síndrome em profissionais mais jovens.

Embora a enfermagem tenha sido classificada pela Health Education Autrority como a quarta profissão mais estressante, são poucas as pesquisas que procuram investigar os problemas associados ao exercício da profissão do Enfermeiro. A história da enfermagem revela que desde sua implementação ela é uma categoria marginalizada e assim, o enfermeiro vem tentando se afirmar profissionalmente sem contar com apoio e compreensão de outros profissionais ${ }^{11}$.

Em suma, o desencadeamento da Síndrome de Burnout, trata-se da ausência de qualidade de vida no trabalho. Evidenciou-se a necessidade da busca por novas formas de gestão, incorporando novos conhecimento e habilidades, sintonizados a uma prática administrativa mais aberta, mais flexível e participativa ${ }^{12}$.

O presente estudo teve por objetivo apontar fatores mais concretos e que podem ajudar a compreender o acometimento da síndrome na prática do Enfermeiro, de modo a contribuir com a discussão e as formas de combate à essa doença, como também incentivar novos estudos para desenvolvimento de ferramentas diagnósticas que auxiliem na gestão dos serviços.

Como sugestão seria interessante ter um suporte para o Cuidado da Saúde Mental dos Profissionais que atuam nos serviços de saúde, preferencialmente aqueles Enfermeiros que estão atuando na Estratégia de Saúde da Família, visto que com essa situação atual econômica no qual estamos passando, a quantidade de pessoas que estão expostas à agravos e procuram os serviços de 
ISSN 2179-6750

saúde estão cada vez maiores e exigindo mais desses profissionais.

Outra sugestão seria uma Ouvidoria específica que pudesse registrar as queixas destes Enfermeiros que atuam na Atenção Básica de Saúde, e com isso mapear os problemas e/ou dificuldades que estão presentes na rotina do trabalho destes profissionais, com isso uma vez identificados, traçar novas condutas e posturas através de um planejamento estratégico assertivo por parte dos gestores locais, melhorando a qualidade de vida no trabalho destes Enfermeiros.

\section{Referências}

1. Trindade LL, Lautert L. Síndrome de burnout entre os trabalhadores da estratégia de saúde da família. Revista Escola de Enfermagem USP, São Paulo. 2010; 44 (2); 274-279.

2. Trigo TR, Teng CT, Hallk JEC. Síndrome de burnout ou estafa profissional e os transtornos psiquiatrícos. Rev. Psiq. Clin, São Paulo. 2007; 34(5); p.223-233.

3. Rossi SS, Santos PG, Passos JP. A síndrome de burnout no enfermeiro: um estudo comparativo entre atenção básica e setores fechados hospitalares. Revista de Pesquisa: Cuidado é fundamental. 2010; 2(4); 1232-1239.

4. Grazziano ES, Bianchi F. Impacto do stress ocupacional. Revista Enferméria Global. Revista Científica da Universidade de Murcia. Espanha. 2010; 01-20.

5. Dias EC. Doenças relacionadas ao trabalho: manual de procedimentos para os serviços de Saúde. Brasília: Ministério da Saúde, 2011. 580 p.

6. Santos M. Por uma outra globalização - do pensamento único à consciência universal. São Pauto: Record, 2000.

7. Lopes CCP, Ribeiro TP, Martinho NJ. Síndrome de burnout e sua relação com a ausência de qualidade de vida no trabalho do enfermeiro. Enfermagem em Foco. 2012; 97-101.

8. Meneguhini F, Paz AA, Lautert L. Fatores ocupacionais associados aos componentes da síndrome de burnout em trabalhadores de enfermagem. Texto Contexto Enfermagem. Florianópolis. 2011; 20 (2); 225-233.

9. Santos PG, Passo JP. A síndrome de burnout e seus fatores desencadeantes em enfermeiros de unidades básicas de saúde. Revista de Pesquisa: Cuidado é fundamental. 2009; 2 (1); 235-240.

10. Ramos ACG. Estresse e a enfermagem na estratégia saúde da família: uma proposta de intervenção. 2014. 38 f. TCC (Graduação) - Curso de Especialização em Atenção Básica em Saúde da Família, Universidade Federal de Minas Gerais, Montes Claros, 2014.

11. Stacciorini JM, Tróccoli BT. O estresse na atividade ocupacional do enfermeiro. Revista 132 
ISSN 2179-6750

Latino-am Enfermagem. 2001; 9(2): 17-25.

12. Matos E, Pires D. Teorias administrativas e organização do trabalho: de Taylor aos dias atuais, influências no setor saúde e na enfermagem. Revista Texto Contexto Enfermagem, 2006; 15(3): 508-14. 